\title{
Efecto de diferentes diseños de drenajes de filtros de arena en la obturación de goteros utilizando aguas residuales regeneradas
}

\author{
C. Solé-Torres ${ }^{1}$, M. Duran-Ros ${ }^{1}$, G. Arbat ${ }^{1}{ }^{,}$J. Pujol $^{1}$, F. Ramírez de Cartagena ${ }^{1}$, J. Puig-Bargués ${ }^{1}$ \\ 1 Departamento de Ingeniería Química, Agraria y Tecnología Agroalimentaria, Escuela Politécnica \\ Superior, Universidad de Girona. C. de M. A. Capmany 61, 17003 Girona; carles.sole@udg.edu; \\ miquel.duranros@udg.edu; gerard.arbat@udg.edu; joan.pujol@udg.edu; francisco.ramirez@udg.edu; \\ jaume.puig@udg.edu
}

\begin{abstract}
Resumen: Los filtros de arena son los que se recomiendan habitualmente cuando se utilizan aguas regeneradas en instalaciones de riego por goteo, si bien se desconoce el efecto de sus distintos diseños de drenaje en la prevención del atascamiento de los goteros. Por este motivo, en este trabajo se ha analizado el efecto en la obturación de goteros provocado por el uso de filtros de arena con distintos modelos de drenaje que operaron con agua residual regenerada durante $1000 \mathrm{~h}$. Al inicio, mitad y final del experimento se determinó el caudal emitido de todos y cada uno de los 226 goteros que había en cada uno de los 12 laterales de riego. Los resultados indican que se produjo una reducción significativa $(\mathrm{p}<0.05)$ del caudal medido del $8 \%$ a las 500 $\mathrm{h}$ y del $11 \%$ a las $1000 \mathrm{~h}$ respecto al inicial. La posición del gotero también tuvo un efecto significativo a partir de las $500 \mathrm{~h}$ de operación. Los caudales de los goteros fueron significativamente inferiores ( $\mathrm{p}<0.05)$ únicamente en los últimos $2 \mathrm{~m}$ de los laterales, hallándose los valores mínimos en los dos últimos goteros. El número de goteros totalmente obturados fue del $1.2 \%$ con el filtro de drenaje de medio poroso, del 1.0\% con el drenaje de cúpulas insertadas y del $0.6 \%$ con el filtro de brazos colectores.
\end{abstract}

Palabras clave: riego por goteo, filtración, filtros granulares, atascamiento.

\section{Introducción}

El uso de aguas residuales regeneradas en agricultura resulta una alternativa viable para hacer frente a las cada vez más comunes situaciones de escasez de agua [1], a la vez que permite liberar aguas de mejor calidad para otros usos [2].

La mejor técnica para la aplicación en riego agrícola de las aguas residuales regeneradas desde un punto de vista sanitario y ambiental es el riego por goteo [3, 4], aunque esta tecnología presenta un problema importante en el atascamiento de los goteros [3, 5]. La obturación de los goteros depende de múltiples factores como el tipo de agua utilizada, el tipo de emisor, las condiciones de mantenimiento y el sistema de filtrado [6, 7]. Los goteros autocompensantes [8, 9], integrados [9] y los de caudales elevados [5, 10] son más resistentes a la obturación.

Existe un amplio consenso en que la filtración es una operación esencial para prevenir la obturación de los goteros [11], aunque no la consigue evitar completamente [12]. Los filtros de arena son los que ofrecen una elevada protección en las instalaciones de riego por goteo [13] ya que consiguen una reducción más eficaz de los sólidos en suspensión presentes en las aguas de riego [7], evitando mejor la obturación [6]. Algunos autores han estudiado la influencia del diseño del drenaje de los filtros de arena en la pérdida de carga $[14,15,16]$, pero ninguno de ellos ha 


\section{CONGRESO IBÉRICO DE AGROINGENIERÍA \\ X CONGRESSO IBÉRICO DE AGROENGENHARIA \\ 3 - 6 septiembre 2019, Huesca - España}

analizado cómo el diseño del filtro afecta a la obturación de los goteros. Por otra parte, los filtros de arena tienen que someterse a contralavados periódicos para liberar las partículas retenidas en el medio si se quiere garantizar su buen funcionamiento [17].

Aunque los procesos de filtración y lavado han sido estudiados [18] pocos son los estudios centrados en intentar mejorar el diseño y funcionamiento de los filtros de arena. En este sentido, Bové et al. (2017) [19] diseñó un nuevo sistema de drenaje para reducir la pérdida de carga a través del lecho filtrante y mejorar la eficiencia de consumo tanto de agua como de energía.

El objetivo de este trabajo fue analizar el efecto de tres filtros de arena con diferentes diseños de drenaje (el prototipo diseñado por Bové et al. (2017) [19] y dos filtros comerciales) en la obturación de los goteros utilizando aguas residuales regeneradas.

\section{Materiales y métodos}

\subsection{Diseño experimental}

Se utilizó el agua residual regenerada de la Estación Depuradora de Aguas Residuales (EDAR) de Celrà (Girona). El sistema experimental consistió en tres filtros de arena con tres drenajes distintos: un filtro modelo FA1M (Lama, Sevilla, España) con drenaje de brazos colectores, otro modelo FA-F2-188 (Regaber, Parets del Vallès, España) con drenaje de cúpulas insertadas y el filtro experimental propuesto por Bové et al. (2017) [19] con drenaje de medio granular. La Figura 1 muestra el diseño de los tres drenajes utilizados.

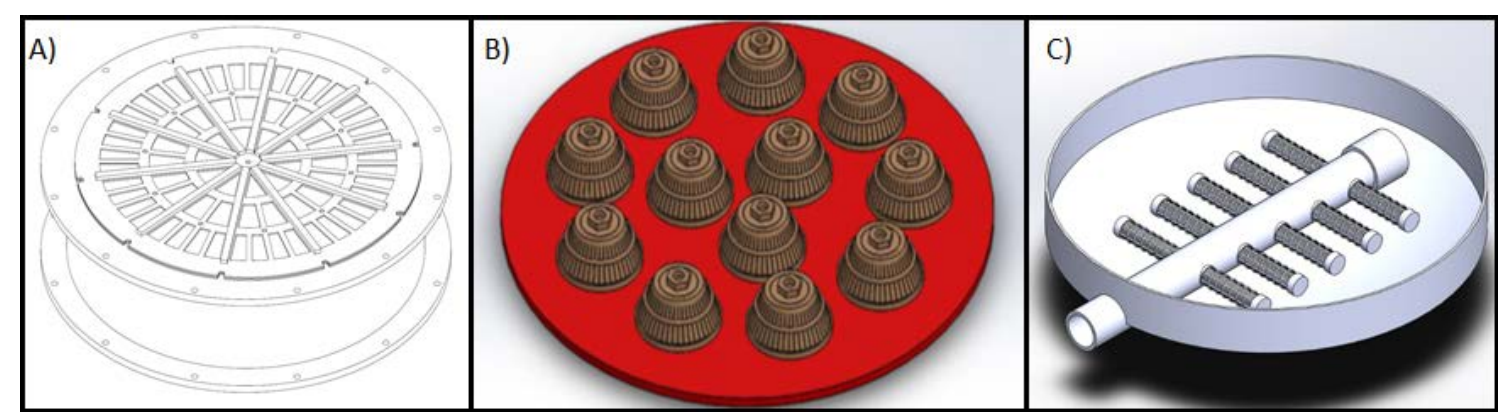

Figura 1. Diseños de drenajes utilizados: medio poroso (A), cúpulas insertadas (B) y brazos colectores (C).

Todos los filtros fueron rellenados con arena silícea con un diámetro efectivo de $0.48 \mathrm{~mm}$ y un coeficiente de uniformidad de 1.73. Cada filtro tenía asociada una subunidad de riego, con 4 laterales de $90 \mathrm{~m}$ cada uno (Figura 2). Cada lateral tenía 226 emisores, con lo que por cada posición de gotero había 12 repeticiones (12 laterales). Sólo en la última posición había 3 goteros por subunidad (9 en total). Se utilizaron goteros autocompensantes Uniram TM AS 16010 (Netafim, Tel Aviv, Israel) de 2.3 1/h de caudal nominal, separados $0.4 \mathrm{~m}$, con un rango de presiones de trabajo entre 50 y $400 \mathrm{kPa}$ y y un coeficiente de variación de fabricación del 3\%.

La instalación disponía de un sistema de impulsión gobernado por un sistema de supervisión, control y adquisición de datos (SCADA), y un variador de frecuencia ajustaba el punto de funcionamiento de la bomba. El caudal de funcionamiento se determinó mediante un caudalímetro electromagnético, que disponía de un transmisor de impulsos con señal 4-20 mA que permitía conocer el caudal exacto de funcionamiento. El sistema experimental permitía sólo el funcionamiento de un filtro a la vez. Puesto que se filtraba más caudal de agua de la necesaria en la subunidad de riego, una válvula proporcional de tres vías conducía el caudal de agua filtrado sobrante hacia un tanque donde se almacenaba, para ser utilizado en los lavados. El agua se cloró después de ser filtrada hasta alcanzar de 2 ppm de cloro libre, valor que se incrementó 


\section{CONGRESO IBÉRICO DE AGROINGENIERÍA \\ X CONGRESSO IBÉRICO DE AGROENGENHARIA \\ 3 - 6 septiembre 2019, Huesca - España}

hasta los 4 ppm cuando se utilizaba para efectuar el lavado de los filtros. Los lavados se activaban cuando la caída de presión sobrepasaba los $50 \mathrm{kPa}$ y tenían una duración de 3 minutos.

Se instalaron sensores para medir la calidad del agua antes y después de ser filtrada. Los parámetros que se midieron antes del proceso de filtración fueron conductividad eléctrica (CE), $\mathrm{pH}$, temperatura, oxígeno disuelto (OD) y turbidez. Los parámetros que se midieron después del proceso de filtración fueron oxígeno disuelto y turbidez.

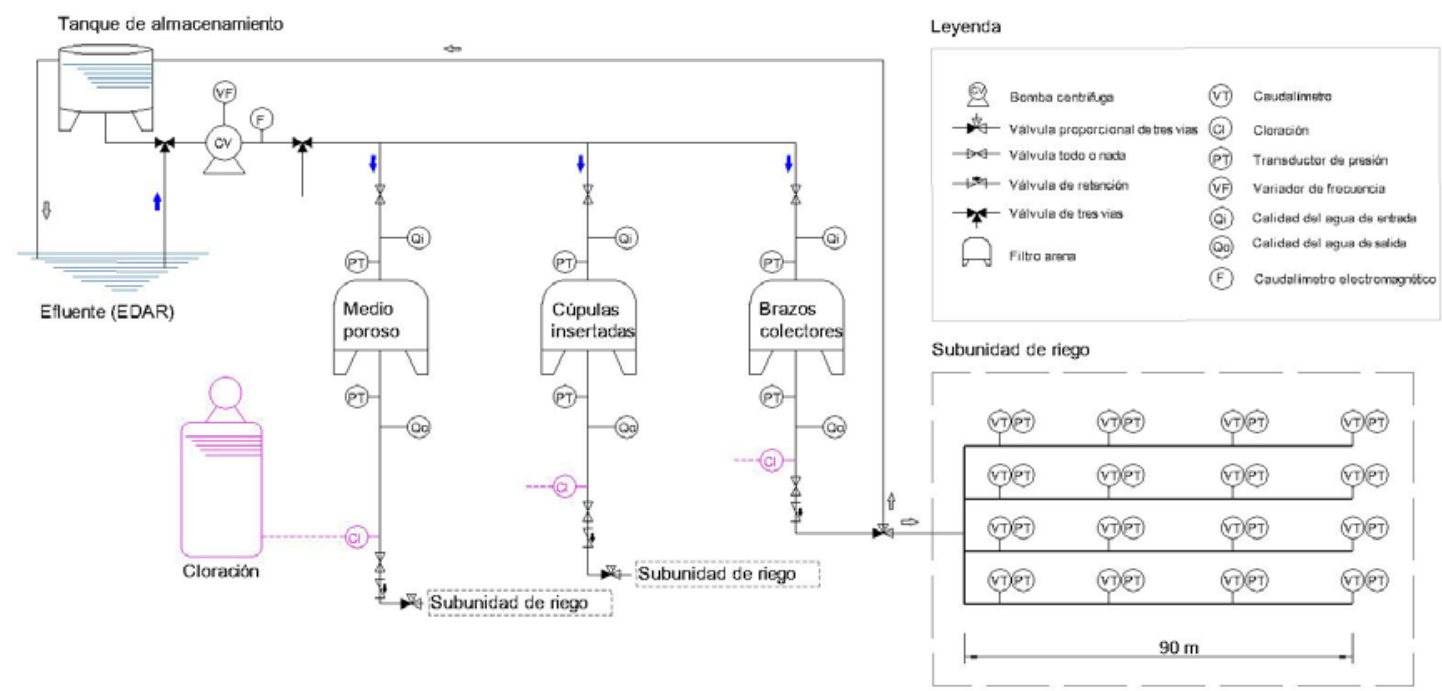

Figura 2. Esquema del diseño experimental.

\subsection{Procedimiento operacional}

El experimento se desarrolló a lo largo de 1000 h, durante las cuales se establecieron dos sesiones diarias de riego de 4 horas por filtro. Durante el experimento, se utilizaron dos alturas de lecho filtrante $(20$ y $30 \mathrm{~cm}$ ) y dos velocidades de filtración $(30$ y $60 \mathrm{~m} / \mathrm{h}$ ) en cada filtro. Cada combinación de altura de lecho filtrante y velocidad de filtración se mantuvo durante $250 \mathrm{~h}$ en todos los filtros. Durante todo el ensayo, no se realizó ningún tipo de limpieza por arrastre de los sedimentos acumulados en los laterales.

\subsection{Evaluación del filtro y los emisores}

La eficiencia de reducción (E) de turbidez y oxígeno disuelto en los filtros fue calculada con la expresión:

$$
E=\frac{N o-N}{N o} \times 100
$$

donde $\mathrm{N}_{0}$ y $\mathrm{N}$ son los valores de dichos parámetros a la entrada y salida de los filtros, respectivamente.

Se midió el caudal de todos los emisores de las subunidades (un total de 2712 emisores) al inicio del experimento $(0 \mathrm{~h})$, a las $500 \mathrm{~h}$ y al final del experimento $(1000 \mathrm{~h})$, así como la presión en cuatro posiciones de cada lateral de riego (al inicio, a 1/3 de la longitud del lateral, a 2/3 y al final). Se pudo calcular así la uniformidad de distribución de presiones UP ${ }_{1 p}[20]$ utilizando la expresión:

$$
U P l q=\left(\frac{\mathrm{p} 25}{\overline{\mathrm{p}}}\right)^{x} \times 100
$$




\section{CONGRESO IBÉRICO DE AGROINGENIERÍA \\ X CONGRESSO IBÉRICO DE AGROENGENHARIA \\ 3 - 6 septiembre 2019, Huesca - España}

donde $\mathrm{p}_{25}$ es la media del $25 \%$ de las localizaciones con menor presión $(\mathrm{kPa}), \overline{\mathrm{p}}$ es la media de todas las presiones de todas las localizaciones muestreadas y $x$ es el coeficiente de descarga.

Al final del ensayo, los emisores de las posiciones 1, 224, 225 y 226 del primer y segundo lateral se abrieron para realizar una inspección visual.

\subsection{Características del agua residual entrante}

La Tabla 1 muestra las medias de los parámetros de calidad del agua entrante durante todo el ensayo. Debido a la heterogeneidad del agua de la estación depuradora, existieron diferencias significativas $(\mathrm{P}<0.05)$ entre algunos de los parámetros de las aguas residuales utilizadas con los distintos filtros.

Tabla 1. Media y error estándar de los parámetros fisicoquímicos del efluente de entrada utilizado en los ensayos. Letras distintas indican que existen diferencias significativas $(\mathrm{P}<0.05)$ en cada parámetro.

\begin{tabular}{cccccc}
\hline $\begin{array}{c}\text { Diseño del drenaje } \\
\text { del filtro }\end{array}$ & $\begin{array}{c}\mathbf{p H} \\
(-)\end{array}$ & $\begin{array}{c}\text { Temperatura } \\
\left({ }^{\circ} \mathrm{C}\right)\end{array}$ & $\begin{array}{c}\mathrm{CE} \\
(\mathrm{dS} / \mathrm{m})\end{array}$ & $\begin{array}{c}\text { DO } \\
(\mathrm{mg} / \mathbf{l})\end{array}$ & $\begin{array}{c}\text { Turbidez } \\
(\text { FNU) }\end{array}$ \\
\hline Medio poroso & $\begin{array}{c}7.33 \pm 0.02 \\
\mathrm{~b}\end{array}$ & $20.61 \pm 0.25 \mathrm{a}$ & $2.64 \pm 0.03 \mathrm{a}$ & $3.27 \pm 0.06 \mathrm{~b}$ & $6.22 \pm 0.16$ \\
Cúpulas insertadas & $\begin{array}{c}7.43 \pm 0.02 \\
\mathrm{a}\end{array}$ & $20.12 \pm 0.22 \mathrm{ab}$ & $2.46 \pm 0.03 \mathrm{~b}$ & $3.57 \pm 0.07 \mathrm{a}$ & $5.82 \pm 0.20$ \\
Brazos colectores & $\begin{array}{c}7.31 \pm 0.02 \\
\mathrm{~b}\end{array}$ & $19.68 \pm 0.25 \mathrm{~b}$ & $2.63 \pm 0.03 \mathrm{a}$ & $3.28 \pm 0.07 \mathrm{~b}$ & $6.42 \pm 0.20$ \\
\hline
\end{tabular}

\subsection{Análisis estadístico}

Para el análisis estadístico se utilizó el programa SPSS Statistics (IBM, New York, Estados Unidos). Para la diferenciación de medias, se utilizó el test de Tukey con un intervalo de confianza del 95\%.

\section{Resultados y discusión}

\subsection{Evaluación de los filtros}

La Tabla 2 muestra las reducciones porcentuales de oxígeno disuelto y turbidez calculadas con la ecuación (1) para los distintos filtros ensayados.

Tabla 2. Media y error estándar de las reducciones de oxígeno disuelto y turbidez logrados por los distintos filtros (\%). Resultados negativos indican un aumento del parámetro. Distintas letras indican que hubo diferencias significativas $(\mathrm{P}<0.05)$ en la reducción del parámetro.

\begin{tabular}{ccc}
\hline \multirow{2}{*}{ Diseño del drenaje del filtro } & \multicolumn{2}{c}{ Reducción (\%) } \\
& Oxígeno disuelto & Turbidez \\
\hline Medio poroso & $-11.20 \pm 2.63$ & $26.29 \pm 1.27 \mathrm{a}$ \\
Cúpulas insertadas & $-6.68 \pm 2.05$ & $18.53 \pm 1.60 \mathrm{~b}$ \\
Brazos colectores & $-11.03 \pm 2.57$ & $13.45 \pm 1.78 \mathrm{~b}$ \\
\hline
\end{tabular}

El filtro con medio poroso mostró una reducción de turbidez (26.29\%) significativamente mayor $(\mathrm{P}<0.05)$ que las conseguidas por el filtro de cúpulas insertadas $(18.53 \%)$ y el de brazos colectores $(13.45 \%)$. Las menores reducciones de turbidez obtenidas con respecto estudios anteriores [7, 18, 21, 22] podrían ser explicadas por los bajos niveles de turbidez del agua de entrada utilizada en el presente ensayo, y por la altura de lecho filtrante fijada en $0.30 \mathrm{~m}$, ya que estaba limitada por el filtro de brazos colectores, cuya altura máxima era de $0.40 \mathrm{~m}$. 


\section{CONGRESO IBÉRICO DE AGROINGENIERÍA \\ X CONGRESSO IBÉRICO DE AGROENGENHARIA \\ 3 - 6 septiembre 2019, Huesca - España}

El filtro con medio poroso obtuvo un mayor aumento de oxígeno disuelto (11.20\%) que el filtro de brazos colectores $(11.03 \%)$ y el filtro de cúpulas insertadas $(6.68 \%)$, aunque sin que hubiera diferencias estadísticamente significativas entre ellas.

\subsection{Distribución de la presión en los laterales.}

El coeficiente de distribución de presiones fue superior al 90\% a lo largo de todo el ensayo, por lo que se pudo considerar uniforme. Como el coeficiente de variación de fabricación de los goteros era inferior al 3\%, las diferencias en el caudal de estos pudieron ser explicadas principalmente por la obturación de los emisores. No se observaron diferencias significativas entre los distintos valores de UPlp entre laterales ni tampoco entre tiempos de muestreo, por lo que no hubo efecto de este factor a lo largo del experimento.

\subsection{Evaluación de los goteros}

El caudal de todos los emisores medido a las 0, 500 y $1000 \mathrm{~h}$ del ensayo se analizó estadísticamente, hallándose un efecto significativo de cada factor fijo (tiempo, posición del gotero y diseño del filtro) así como las interacciones de diseño y tiempo, diseño y posición y tiempo y posición (todas ellas con un $\mathrm{P}<0.001$ ). En términos generales, se constató una caída del caudal de los goteros del $10.80 \%$ desde el momento inicial hasta el final del experimento (de 2.49 1/h de caudal medio al inicio hasta 2.22 l/h transcurridas 1000 h). Esta reducción de caudales a lo largo del tiempo debido a la incidencia de las obturaciones ha sido ampliamente observada por diversos autores $[5,7,9,21,22]$.

\subsubsection{Efecto del diseño del filtro y el tiempo de riego}

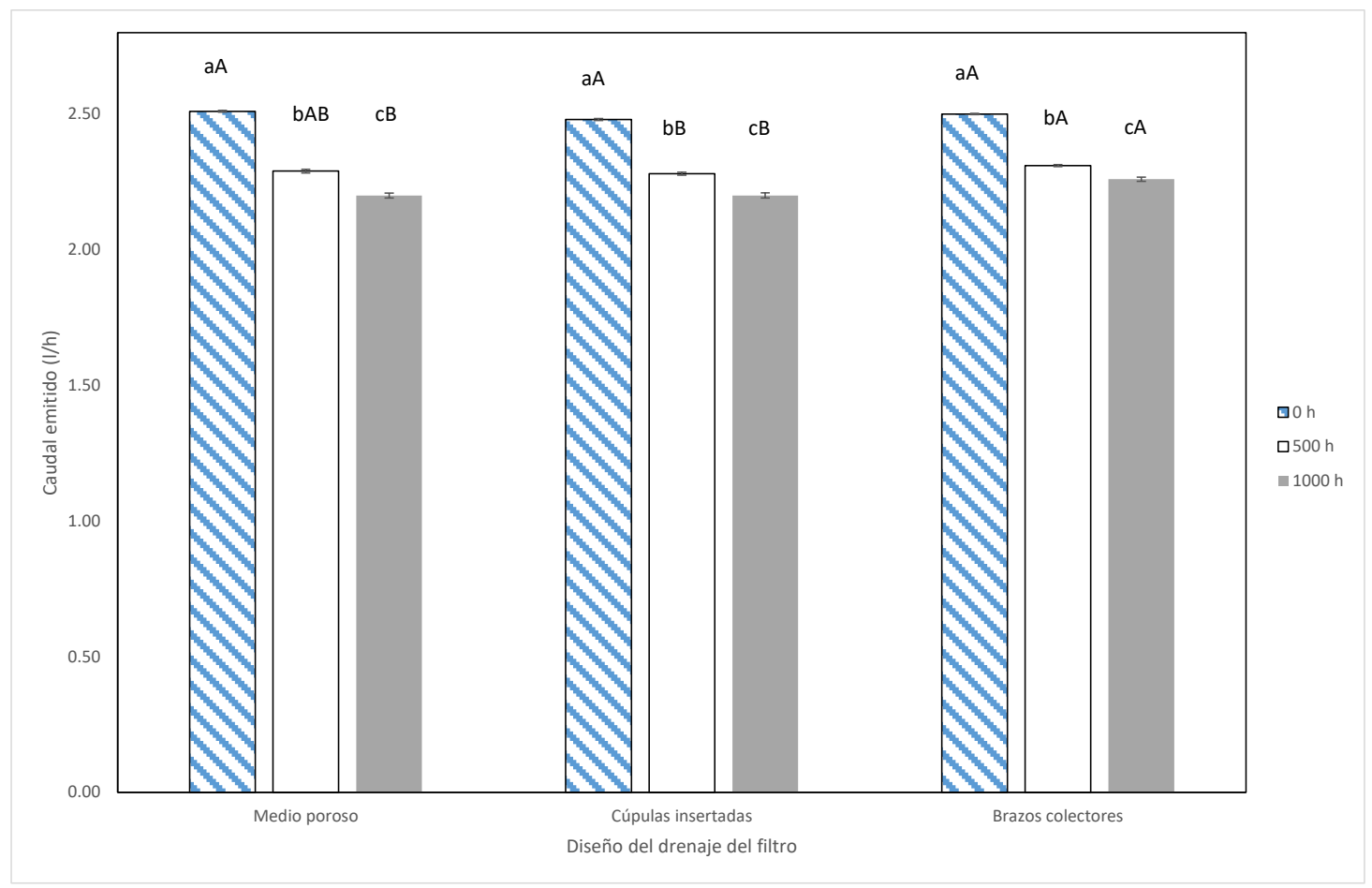

Figura 3. Caudal medio y error estándar $(1 / h)$ de todos los goteros para cada tipo de diseño de drenaje del filtro de arena, en los tres tiempos distintos de medición. Para cada diseño de drenaje, letras minúsculas distintas indican diferencias significativas entre tiempos $(\mathrm{P}<0.05)$. Para cada tiempo medido, letras mayúsculas distintas indican diferencias significativas $(\mathrm{P}<0.05)$ entre los diseños de filtros. 


\section{CONGRESO IBÉRICO DE AGROINGENIERÍA \\ X CONGRESSO IBÉRICO DE AGROENGENHARIA \\ 3 - 6 septiembre 2019, Huesca - España}

La Figura 3 muestra el caudal de los goteros en función del diseño del drenaje del filtro de arena y la duración del ensayo. Hubo una reducción significativa del caudal a lo largo del tiempo de funcionamiento para cada diseño, siendo menor en el filtro de brazos colectores (del $7.6 \%$ a las $500 \mathrm{~h}$ y del $9.6 \%$ a las $1000 \mathrm{~h}$ ) frente a los 8.76 y $8.06 \%$ obtenidos por el filtro de medio poroso y del 12.35 y $11.29 \%$ por el filtro de cúpulas insertadas, a las 500 y $1000 \mathrm{~h}$ respectivamente. La reducción del caudal fue mayor en las primeras $500 \mathrm{~h}$ que en las siguientes 500 , hecho también referenciado en la bibliografía [22]. Al principio del ensayo ( $0 \mathrm{~h})$ no se encontraron diferencias significativas. Pasadas $500 \mathrm{~h}$, el caudal de los goteros del filtro de brazos colectores fue significativamente mayor $(2.31 \mathrm{l} / \mathrm{h})$ que con el filtro de cúpulas insertadas $(2.28 \mathrm{l} / \mathrm{h})$ pero no que con el de medio poroso (2.29 l/h). Sin embargo, pasadas $1000 \mathrm{~h}$, el caudal medio de los goteros del filtro de brazos colectores fue significativamente mayor $(2.26 \mathrm{l} / \mathrm{h})$ que el de medio poroso y cúpulas insertadas (ambos con $2.20 \mathrm{l} / \mathrm{h}$ ). Asimismo, de promedio, estas diferencias sólo representaron el 3\% del caudal de los emisores.

\subsubsection{Efecto del diseño del drenaje del filtro y la posición del gotero.}

La Figura 4 muestra los caudales respecto el diseño del filtro y la ubicación de los goteros. Para cada diseño de filtro, se hallaron diferencias significativas en los caudales, pero únicamente para los localizados al final del lateral. Tanto para el drenaje de medio poroso como para el de cúpulas insertadas, el caudal de los tres últimos goteros (últimos $1.2 \mathrm{~m}$ del lateral) fue significativamente $(\mathrm{P}<0.05)$ inferior que el de los goteros del resto del lateral (esto es, de las posiciones 1 a 222). En cambio, para el diseño de brazos colectores, sólo el caudal de los dos últimos emisores fue significativamente inferior al del resto. Para todos los tres diseños de drenajes estudiados, el último emisor obtuvo claramente el menor caudal medio.

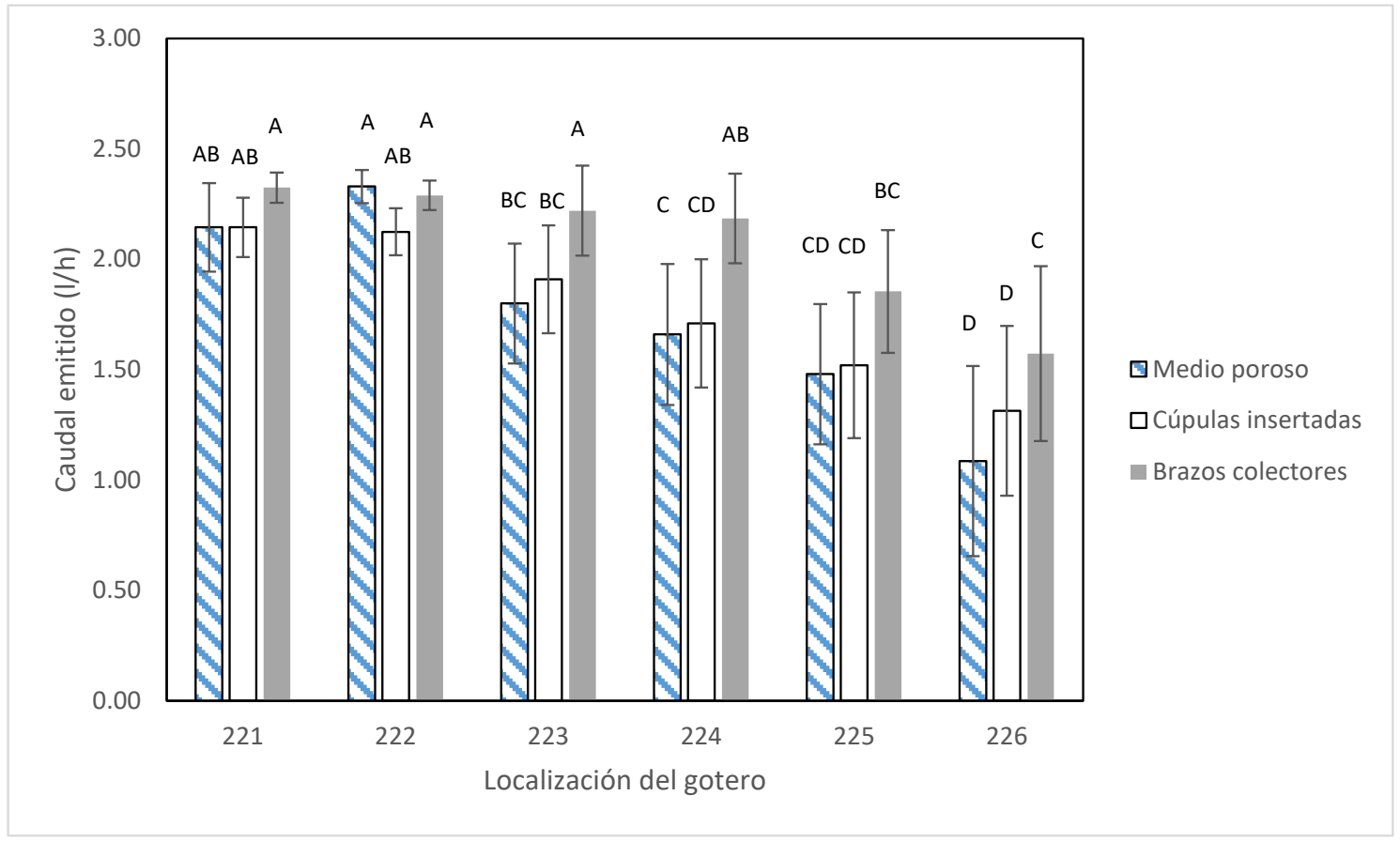

Figura 4. Media del caudal y error estándar $(1 / h)$ de los 6 últimos goteros del lateral para cada diseño de drenaje. Para cada diseño, letras distintas indican diferencias significativas $(\mathrm{P}<0.05)$ entre localizaciones. 


\section{CONGRESO IBÉRICO DE AGROINGENIERÍA \\ X CONGRESSO IBÉRICO DE AGROENGENHARIA \\ 3 - 6 septiembre 2019, Huesca - España}

Para cada localización de los goteros, únicamente se encontraron diferencias en el caudal emitido de 11 goteros entre diseños de filtros, representando el $5 \%$ de los emisores de cada lateral. Además, las localizaciones fueron muy dispersas y no seguían ningún patrón.

\subsubsection{Efecto del tiempo y la localización de los emisores}

La interacción entre tiempo y ubicación del gotero resultó significativa $(\mathrm{P}<0.05)$. Al principio del ensayo, no hubo diferencias entre localizaciones (Figura 5), mientras que pasadas $500 \mathrm{~h}$ los tres últimos emisores (posiciones 224, 225 y 226) emitieron significativamente menor caudal que el resto de los goteros $(1.83,1.83$ y $1.49 \mathrm{l} / \mathrm{h}$, respectivamente). Al cabo de $1000 \mathrm{~h}$, los cuatro últimos emisores (localizaciones 223, 224, 225 y 226) liberaron significativamente menor caudal $(1.45,1.22$, 0.61 y $0.00 / \mathrm{h}$, respectivamente) que el resto de los goteros del lateral. El último gotero (posición 226) se encontró completamente obturado en todas las subunidades de riego.

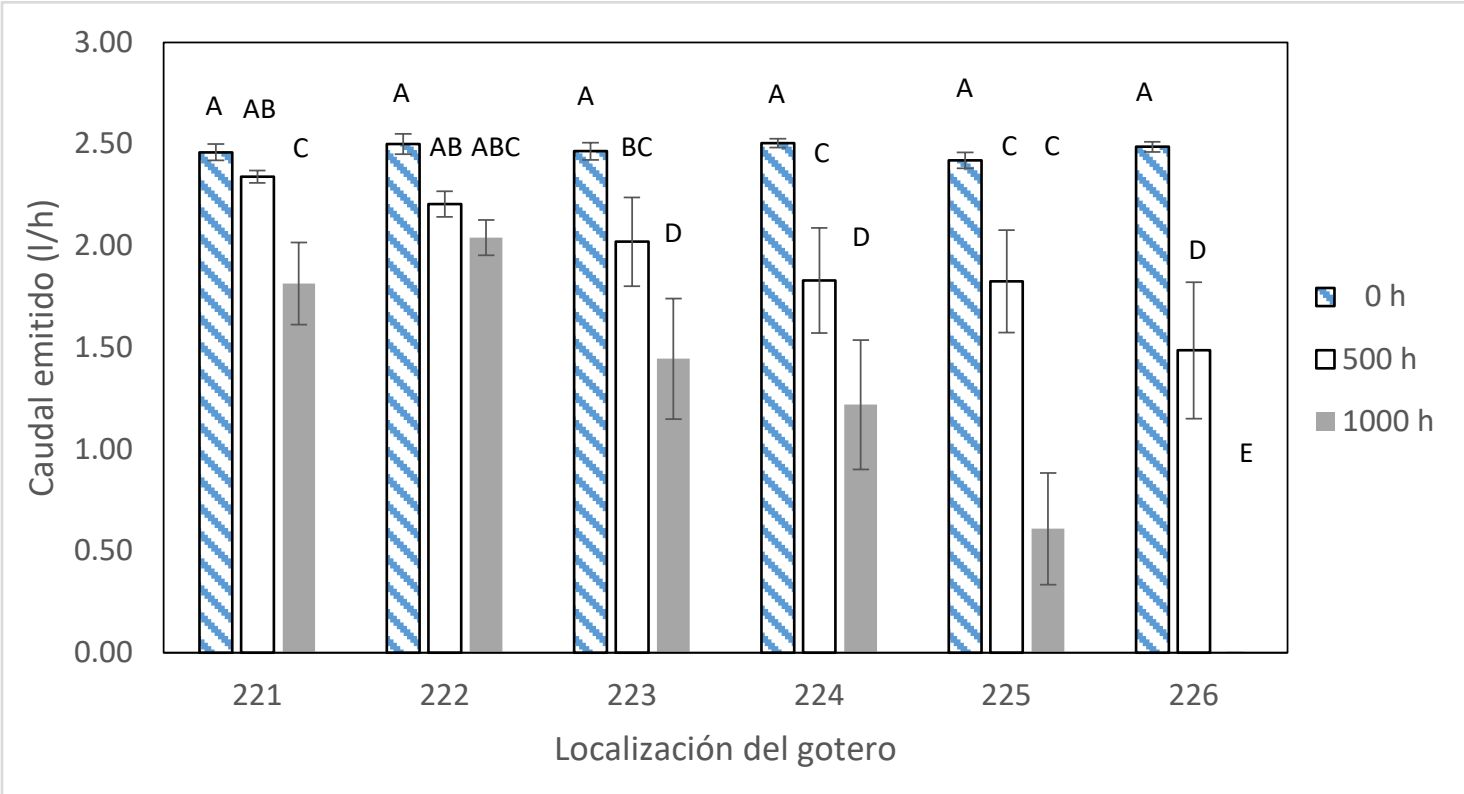

Figura 5. Medias del caudal y el error estándar ( $(1 / \mathrm{h})$ de los 6 últimos goteros, después de 500 y $1000 \mathrm{~h}$ de riego. Para cada tiempo medido, letras distintas indican diferencias significativas $(\mathrm{P}<0.05)$ entre localizaciones.

\subsection{Goteros completamente obturados}

A las $500 \mathrm{~h}$ de ensayo se encontraron 5 goteros completamente obturados en los laterales protegidos por el filtro del drenaje de medio poroso ( $0.56 \%$ del total), 2 para el de cúpulas insertadas $(0.22 \%$ del total) y ninguno en el drenaje de brazos colectores. Transcurridas $1000 \mathrm{~h}$, se observaron 10 goteros completamente obturados en los laterales protegidos por el drenaje de medio poroso ( $1.13 \%$ del total), 8 para el de cúpulas insertadas $(0.91 \%$ del total) y 6 para el de brazos colectores $(0.68 \%$ del total).

Analizando el porcentaje de goteros completamente obturados, se hallaron diferencias significativas entre tiempo, localización y ambos factores, pero no en el diseño del drenaje como factor simple ni en interacción entre tiempo y localización. Esta última afirmación significa que los diferentes diseños del filtro ensayados no afectan el porcentaje de los emisores completamente obturados. A las $500 \mathrm{~h}$, hubo diferencias significativas en el porcentaje de goteros completamente obturados en las localizaciones 223, 224, 225 y 226 respecto el resto de los emisores (Figura 6). Pasadas 1000 h, en la localización 226 se observó significativamente más porcentaje de emisores completamente obturados que en la localización 225, que a su vez fue mayor que las 


\section{CONGRESO IBÉRICO DE AGROINGENIERÍA \\ X CONGRESSO IBÉRICO DE AGROENGENHARIA \\ 3 - 6 septiembre 2019, Huesca - España}

localizaciones 224 y 223 y con menor porcentaje de goteros completamente obturados estuvo la posición 222.

Todos los goteros completamente obturados se encontraron en posiciones del final del lateral. Son varios los autores que han observado la misma tendencia en las obturaciones de los emisores al final de los laterales [5, 8, 13, 22], que podrían atribuirse a la reducción del caudal del emisor al final del lateral [23] y a una mayor concentración de partículas en estas posiciones [22].

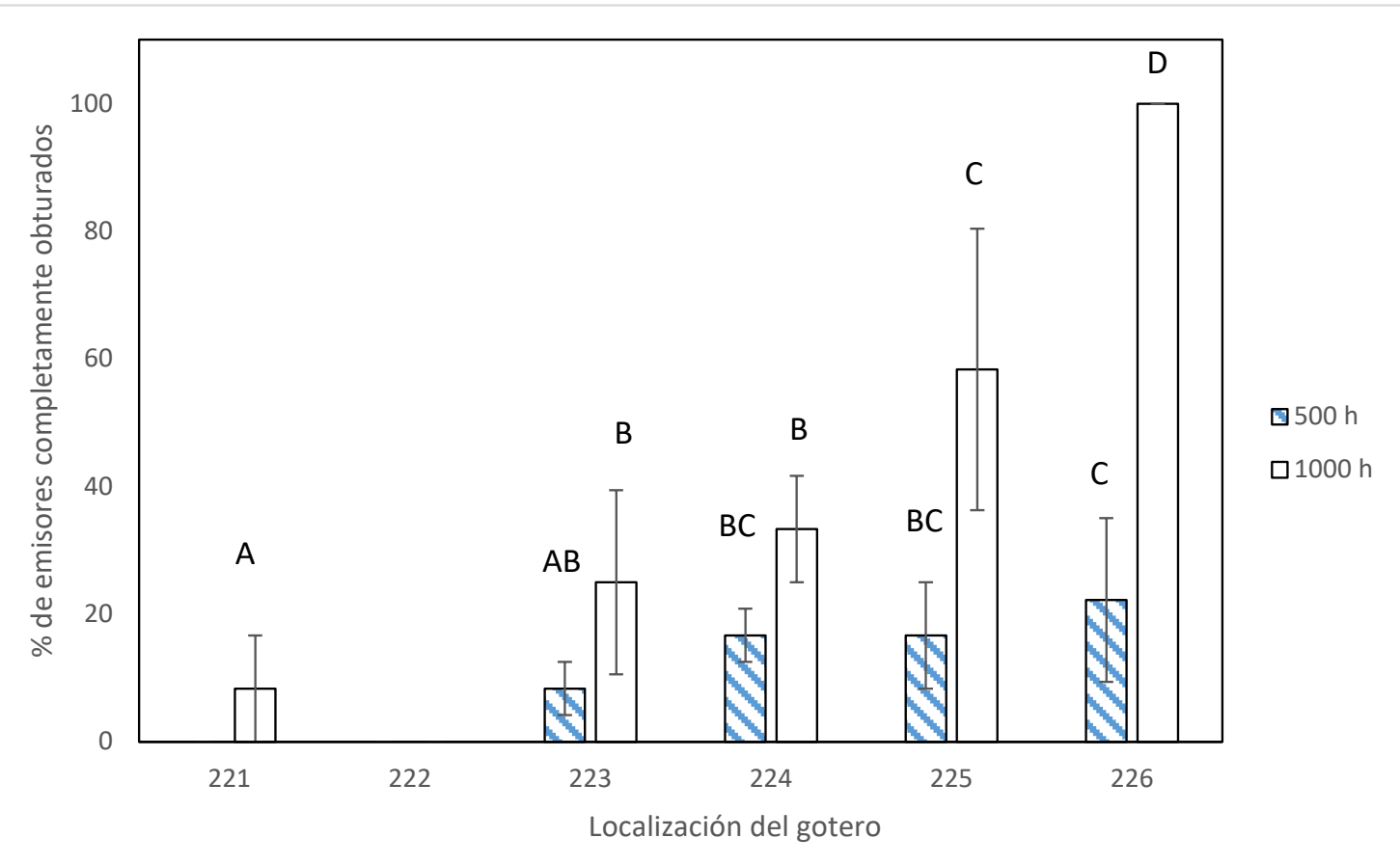

Figura 6. Porcentaje de goteros completamente obturados (\%) por localización de los últimos emisores, después de 500 y 1000 h de ensayo. Para cada tiempo, diferentes letras significan diferencias significativas $(\mathrm{P}<0.05)$ entre localizaciones.

\subsection{Observación de los goteros}

Se observaron algunos goteros una vez finalizado el ensayo. Si bien no se encontraron deposiciones en los goteros ubicados al inicio del lateral (Fig. 7A) para ninguna subunidad de riego, se detectaron crecimientos de películas microbianas (biofilm) y sedimentos en los últimos goteros de todas las subunidades de riego (Fig. 7B), así como crecimiento de biofilm en el orificio de salida de los emisores (Fig. 7C), no determinándose diferencias visuales entre subunidades. Estos resultados concuerdan con los hallazgos de varios autores $[5,7,8,10]$. 


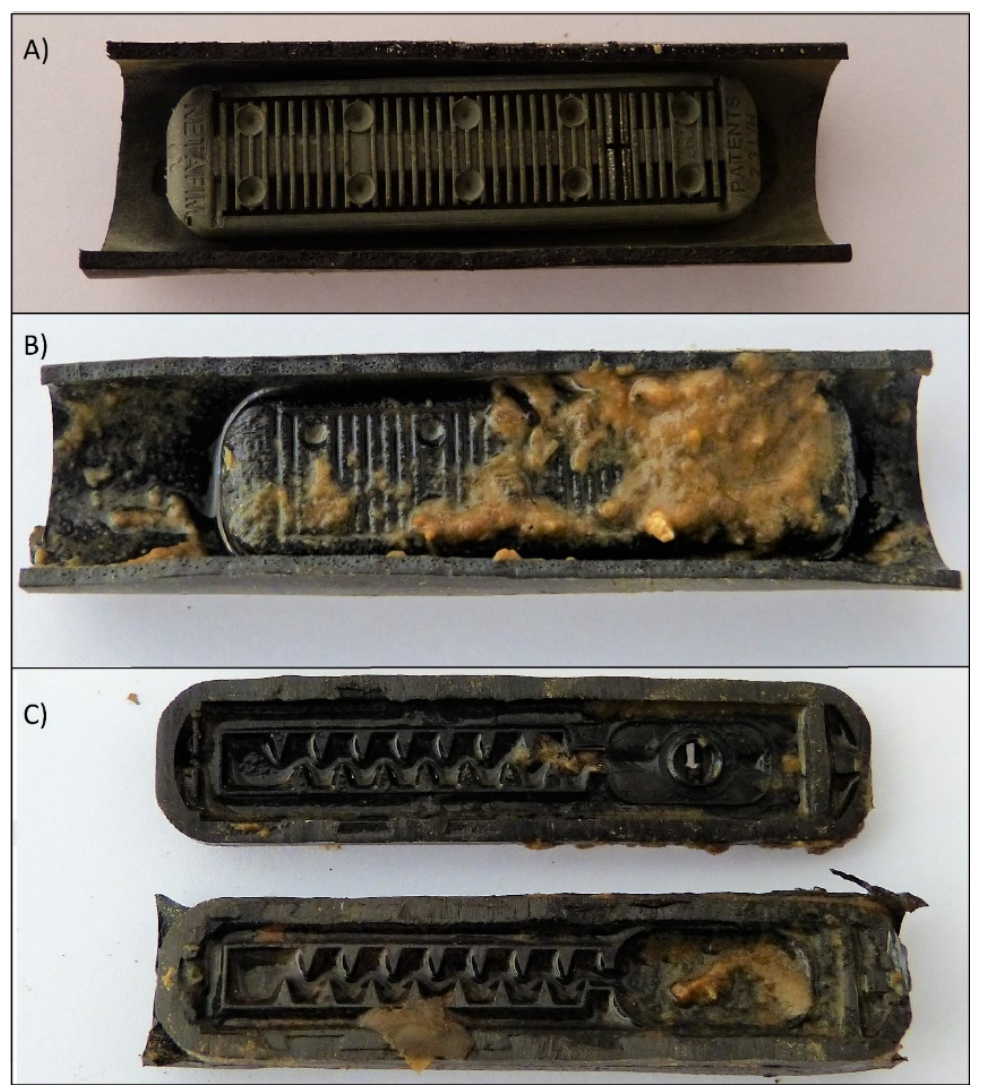

Figura 7. Vista exterior de los goteros protegidos por el diseño de medio poroso en la primera localización (A) y en la última (B), y vista interior de este último emisor (C) pasadas las $1000 \mathrm{~h}$ de ensayo.

\section{Conclusiones}

El presente estudio fue llevado a cabo para determinar el efecto del diseño del drenaje de tres filtros de arena en la obturación de los goteros utilizando agua residual regenerada clorada. El tiempo de ensayo fue de $1000 \mathrm{~h}$ y la longitud de los laterales $90 \mathrm{~m}$.

La obturación de los emisores fue debida a las interacciones entre diseño del drenaje, la localización de los emisores y el tiempo de riego. La localización de los goteros tuvo un efecto significativo sólo después de $500 \mathrm{~h}$ de riego, con caudales significativamente inferiores en los 3 últimos emisores, y después de $1000 \mathrm{~h}$, con caudales significativamente inferiores en los 5 últimos. También se observó una reducción significativa del caudal de los emisores (del 9.60 al 12.35\%) para todas las subunidades de riego después de $1000 \mathrm{~h}$. Los goteros protegidos por el diseño de brazos colectores emitieron más caudal medio que los otros diseños, aunque no fue este el diseño de filtro que retuvo más turbidez.

Por otro lado, el porcentaje de goteros completamente obturados dependió de la interacción entre el tiempo de riego y la localización del gotero, sin que se hallasen diferencias significativas entre los diseños de drenajes.

Finalmente, el filtro con el drenaje de brazos colectores presentó menor obturación utilizando aguas residuales, aunque sólo después de 1000 h de riego. Para tiempos más cortos, la protección frente a la obturación no fue distinta entre los distintos filtros ensayados. Sería recomendable continuar estudiando si los diferentes diseños de drenajes tienen un efecto específico en cada uno de los agentes que participan en la obturación de los emisores. 


\section{CONGRESO IBÉRICO DE AGROINGENIERÍA \\ X CONGRESSO IBÉRICO DE AGROENGENHARIA \\ 3 - 6 septiembre 2019, Huesca - España}

\section{Agradecimientos}

Los autores quieren expresar su agradecimiento al Ministerio de Economía y Competitividad por su financiación a través del proyecto de invetigaciónAGL2015-63750-R. Carles Solé-Torres es el beneficiario de beca pre-doctoral IFUdG2016/72 concedida por la Universidad de Girona. Los autores también quieren agradecer al Ayuntamiento de Celrà por su ayuda en llevar a cabo este experimento.

\section{Referencias}

1. Asano T., Burton F.L., Leverenz H.L. Water reuse: issues, technologies and applications. 2007, McGraw Hill Inc., New York.

2. Lazarova V., Asano T. Challenges of sustainable irrigation with recycled water. In: Lazarova V., Bahri A., Water reuse for irrigation - Agriculture, landscape and Turf grass. 2005, CRC Press, Washington D.C., USA.

3. Bucks D.A., Nakayama F.S., Gilbert R.G. Trickle irrigation water quality and preventive maintenance. Agric. Water Manage. 1979, 2 (2), 149-162.

4. World Health Organization WHO. Guidelines for the safe use of wastewater, excreta and greywater, vol II. 2006, WHO Press, Geneva.

5. Ravina I., Paz E., Sofer Z., Marcu A., Shisha A., Sagi G. Control of emitter clogging in drip irrigation with reclaimed wastewater. Irrig. Sci. 1992, 13 (3), 129-139.

6. Capra A., Scicolone B. Recycling of poor quality urban wastewater by drip irrigation systems. J. Clean. Prod. 2007, 15 (16), 1529-1534.

7. Duran-Ros M., Puig-Bargués J., Arbat G., Barragán J., Ramírez de Cartagena F. Effect of filter, emitter and location on clogging when using effluents. Agric. Water Manage. 2009, 96 (10), 67-79.

8. Puig-Bargués J., Arbat G., Elbana M., Duran-Ros M., Barragán J., Ramírez de Cartagena F., Lamm F.R., Effect of flushing frequency on emitter clogging in microirrigation with effluents. Agric. Water Manage. 2010, 97, 883-891.

9. Pei Y.T., Li Y.K., Liu Y.Z., Zhou B., Shi Z., Jiang Y.G. Eight emitters clogging characteristics and its suitability evaluation under on-site reclaimed water drip irrigation. Irrig. Sci. 2014, 32(2), 141-157.

10. Trooien T.P., Lamm F.R., Stone L.R., Alam M., Rogers D.H., Clark G.A., Schlegel A.J. Subsurface drip irrigation using ivestock wastewater: dripline flow rates. Appl. Eng. Agric. 2000, 16 (5), 505-508.

11. Oron G., Shelef G. Turzynski, B. Trickle irrigation using treated wastewaters. J. Irrig. Drain. Div. 1979, 105 (IR2), 175-186.

12. Tajrishy M.A., Hills D.J., Tchobanoglous G. Pretreatment of secondary effluent for drip irrigation. J. Irrig. Drain. Engrg. 1994, 120 (4), 716-731.

13. Trooien T.P. Hills, D.J. Application of biological effluent. In: Lamm, F.R., Ayars, J.E., Nakayama, F.S. (Eds.), Microirrigation for Crop Production. Design, Operation, and Management. 2007, Elsevier, Amsterdam, pp. 329-356.

14. Mesquita M., Testezlaf R., Ramirez J. The effect of media bed characteristics and internal auxiliary elements on sand filter head loss. Agric. Water Manage. 2012, 115, 178-185.

15. Bové J., Arbat G., Pujol T., Duran-Ros M., Ramírez de Cartagena F., Velayos J., Puig-Bargués J. Reducing energy requirements for sand filtration in microirrigation: improving the underdrain and packing. Biosyst. Eng. 2015, 140, 67-78.

16. Pujol T., Arbat G., Bové J., Puig-Bargués J., Duran-Ros M., Velayos J., Ramírez de Cartagena F. Effects of the underdrain design on the pressure drop in sand filters. Biosyst. Eng. 2016, 150, 1-9.

17. Nakayama F.S., Boman B.J., Pitts D.J. Maintenance. In: Lamm, F.R., Ayars, J.E., Nakayama, F.S. (Eds.), Microirrigation for Crop Production. Design, Operation, and Management. 2007, Elsevier, Amsterdam, pp. 389-430.

18. Elbana M., Ramírez de Cartagena F., Puig-Bargués J. Effectiveness of sand media filters for removing turbidity and recovering dissolved oxygen from a reclaimed effluent used for micro-irrigation. Agric. Water Manage. 2012, 111, 27-33. 


\section{CONGRESO IBÉRICO DE AGROINGENIERÍA \\ X CONGRESSO IBÉRICO DE AGROENGENHARIA \\ 3- 6 septiembre 2019, Huesca - España}

19. Bové J., Puig-Bargués J., Arbat G., Duran-Ros M., Pujol T., Pujol J., Ramírez de Cartagena, F. Development of a new underdrain for improving the efficiency of microirrigation sand media filters. Agric. Water Manage. 2017, 179, 296-305.

20. Bliesner R.D. Field evaluation of trickle irrigation efficiency, in: Proceedings of Water Management for Irrigation and Drainage ASCE. 1976, pp. 382-393.

21. Triphati V.K., Rajput T.B.S., Patel N. Performance of different filter combinations with surface and subsurface drip irrigation systems for utilizing municipal wastewater. Irrig. Sci. 2014, 32, 379-391.

22. Wu W.Y., Huang Y., Liu H.L., Yin S.Y., Niu Y. Reclaimed water filtration efficiency and drip irrigation emitter performance with different combinations of sand and disc filters. Irrig. and Drain. 2015, 64, 362-369.

23. Shannon W.M., James L.G., Basset D.L., Mih W.C. Sediment transport and deposition in trickle irrigation laterals. Trans. ASAE. 1982, 25 (1), 160-164. 